\title{
МЕТОД РАСПРЕДЕЛЕНИЯ ОГРАНИЧЕНИЙ ПОТРЕБЛЯЕМОИ МОЩНОСТИ ПО ОТРАСЛЯМ ПРОМЫШЛЕННОСТИ
}

\section{K. JAANIMAGI. TOOSTUSHARUDEVAHELISE TARBIMISVOIMSUSTE PIIRAMISE JAOTAMISE MEETOD}

K. JAANIMAGI. A METHOD OF DISTRIBUTION OF THE LIMITS OF CONSUMED POWER BETWEEN BRANCHES OF INDUSTRY

\section{(Представлена И. Эпиком)}

Введение ограничений электрической нагрузки в энергосистеме может быть вызвано рядом причин: аварийными ситуациями, недостатком генерирующей пиковой мощности, нехваткой дефицитных видов топлива и т. д. Основным способом регулирования нагрузки в энергосистеме является ее распределение по промышленным предприятиям.

Под оптимальным распределением ограничений потребляемой мощности по отраслям будем понимать такое распределение, при котором экономические, технические и социальные отрицательные последствия для народного хозяйства в целом сводятся до минимума. Введем в рассмотрение шесть целей:

$$
\begin{gathered}
\Phi_{1}=\sum_{i=1}^{N} c_{1 i} x_{i}^{\text {Bap }} \rightarrow \max , \\
\Phi_{2}=\sum_{i=1}^{N} c_{2 i} x_{i}^{\text {Bap }} \rightarrow \min , \\
\Phi_{3}=\sum_{i=1}^{N} \frac{W_{i}^{\text {ycr }}}{W_{i}^{\text {max }}} / \frac{x_{i}^{\text {Bap }}}{x_{i}^{\text {max }}} \rightarrow \min , \\
\Phi_{4}=\sum_{i=1}^{N}\left(1 / c_{3 i}\right)_{i}^{\text {Bap }} \rightarrow \min , \\
\Phi_{5}=\sum_{i=1}^{N} c_{5 i} / \frac{x_{i}^{\text {Bap }}}{x_{i}^{\text {max }}} \rightarrow \min , \\
\Phi_{6}=\sum_{i=1}^{N} c_{6 i} / \frac{x_{i}^{\text {Bap }}}{x_{i}^{\text {max }}} \rightarrow \min ,
\end{gathered}
$$


где $W_{i}^{\text {уст }}, W_{i}{ }^{\text {max }}$ - установленная и максимальная мошности $i$-й отрасли; $c_{1 i}$ - удельная валовая продукция $i$-й отрасли, $p y б / \kappa B T \cdot \vartheta ; c_{2 i}-$ удельный ущерб от введения ограничения на потребление мощности в $i$-й отрасли, $р у б / \kappa B т \cdot 4 ; \quad c_{3 i}$ - электроэнерговооруженность труда, $\kappa B T \cdot 4 / 4 е л ; c_{5 i}, c_{6 i}$ - часы использования максимальной мощности в сутки и в год $i$-й отраслью; $x_{i}^{\text {вар }}, x_{i}^{\max }$ - выделяемое в данном варианте и заданное максимальное количество электроэнергии для $i$-й отрасли; $N$ - количество отраслей промышленности.

Для совокупности отраслей должны выполняться следующие соотношения

$$
\begin{gathered}
\sum_{i=1}^{N} x_{i}=X, \\
x_{i} \leqslant Q_{i},
\end{gathered}
$$

где $X$ - суммарное для всех отраслей количество выделяемой электроэнергии, $Q_{i}$ - потребность в электроэнергии $i$-й отрасли. При решении практической задачи потребность в электроэнергии определялась нами через максимальные мощности за 2 и.

В основу метода для решения задачи (1)-(8) были положены результаты [ $\left.{ }^{1}\right]$. Метод состоит из 3 этапов. Первый этап - сведение целевых функций $\Phi_{1}-\Phi_{6}$ известным преобразованием $\left[{ }^{2}\right]$ к безразмерным, подлежащим минимизации величинам.

Второй этап - выбор $N_{1}$ компромиссных решений из шести областей Парето многоцелевых задач вида

$$
\begin{gathered}
c_{1 i} x_{i} \rightarrow \max , \quad i=\overline{1, N}, \\
\sum_{i=1}^{N} x_{i}=X, \\
x_{i} \leqslant Q_{i},
\end{gathered}
$$

во-первых, на основе критерия наименьшего удаления от идеальных решений $\left.{ }^{3}\right]$ задач типа (9) и, во-вторых, случайным образом.

Третий этап - выбор из компромиссных решений задачи (1)-(8) оптимального. Для этого рассматривается матричная игра с платежной матрицей вида

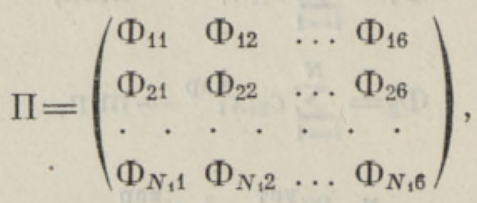

где $\Phi_{k j}-$ значение $j$-го критерия в точке $\bar{x}_{k}=\left(x_{1}{ }^{k}, x_{2}{ }^{k}, \ldots x_{N}{ }^{k}\right)$. Столбцам матрицы соответствуют целевые функции $\Phi_{1}, \Phi_{2}, \ldots, \Phi_{6}$, а строкам - стратегии $\bar{x}_{k}, k=\overline{1, N_{1}}$. Второй игрок при выборе своей оптимальной смешанной стратегии стремится сделать минимальными значения критериев по отношению ко всем $N_{1}$ стратегиям, что является искомым требованием для определения весовых коэффициентов в глобальном критерии

$$
\Phi(\bar{x})=\sum_{i=1}^{6} \lambda_{i} \Phi_{i}(\bar{x})
$$

Оптимальным компромиссным решением задачи (1)-(8) будет решение, доставляющее минимальное значение критерию (10). Весо- 
вые коэффициенты получаются в результате решения задачи линейного программирования, соответствующей задаче второго игрока в рассмотренной матричной игре.

Для решения исходных задач может быть полезной и модификация рассмотренного метода, основанная на решении матричной игры с платежной матрицей $\Pi_{1}$

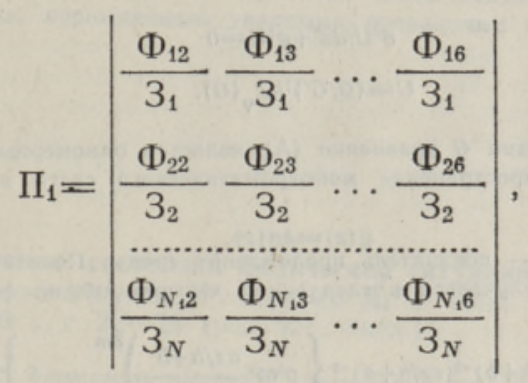

где $3_{i}-$ значение целевой функции $\Phi_{1}$ в стратегии $\bar{x}_{i}$, т. е. суммарная продукция, произведенная $N$ отраслями при соответствующем вектору $\bar{x}_{i}$ распределении ограничений на потребление мощности, руб. Перевод полученных в процессе решения весовых коэффициентов $\lambda_{i}$ в экономические оценки важности целей отразит ожидаемый эффект от принятия той или иной стратегии [ $\left.{ }^{4}\right]$.

В настоящей работе, по-видимому, впервые предпринята попытка решить поставленную задачу формализованным методом. И хотя этот метод имеет ряд недостатков (основной из которых - сложный процесс выбора $N_{1}$ стратегий), он показал хорошую работоспособность при расчете распределения ограничений на потребление мощности и электроэнергии по 10 отраслям промышленности Эстонской ССР. Составленные программы были реализованы на ЭВМ ЕС-1022.

Автор выражает благодарность М. Мытусу за постановку проблемы, внимание и поддержку.

\section{ЛИТЕРАТ У РА}

1. Ю т л е р Х., Экономика и матем. методы, 111, вып. 3, 397-406 (1967).

2. О ко ро ко в В. Р., Управление электроэнергетическими системами, Л., Изд. Лгу, 1976.

3. Я а н и м я г и К., Изв. АН ЭССР, Физ’. Матем., 28, № 1, 80-82 (1979).

4. Долгов П. П., Дан илин О. Е., В кн.: Учет неопределенности исходной информации, прн оптимизащии энергетического хозяйства экономического района, Таллин, Изд. АН ЭССР, 1978, с. 3-20.

Институт термофизики и электрофизики " Академии наук Эстонской ССР
Поступила в редакцию 20/VIII 1979 